\title{
Comparison of Different Methods to Isolate Urinary Extracellular Vesicles Useful as Possible Kidney Damage Biomarkers
}

\section{Andrea Carraro ${ }^{1 *}$, Susanna Negrisolo ${ }^{1}$, Diana Marzenta ${ }^{1}$, Michele Grassi ${ }^{2}$, Anna Maria Tolomeo ${ }^{2}$, Piera De Gaspari $^{3}$, Maurizio Muraca ${ }^{2}$, Giorgio Perilongo ${ }^{2}$ and Luisa Murer ${ }^{1}$}

\author{
${ }^{1}$ Laboratory of Immunopathology and Molecular Biology of the Kidney of Pediatric Nephrology, Dialysis and Transplant Unit, Department of \\ Women's and Children's Health, University-Hospital of Padua, Italy \\ ${ }^{2}$ Department of Women's and Children's Health, Hospital-University of Padua, Italy \\ ${ }^{3}$ Neuroimmunology Laboratory, IRP Città Della Speranza, Padua, Italy \\ *Corresponding Author: Andrea Carraro, Laboratory of Immunopathology and Molecular Biology of the Kidney of Pediatric Nephrology, \\ Dialysis and Transplant Unit, Department of Women's and Children's Health, University-Hospital of Padua, Italy.
}

Received: October 09, 2019; Published: October 22, 2019

DOI: $10.31080 /$ ASMS.2019.03.0446

\begin{abstract}
Extracellular vesicles are lipid membrane-bound nanoparticles released from cells in different biological fluids. Particularly, urinary extracellular vesicles (UEVs) arising from the nephron cells, could be helpful as novel biomarkers for kidney allograft injury. This comparative study aims to identify the most efficient UEVs isolation method to detect novel kidney biomarkers. UEVs were isolated from urine samples using four commercial kits and purified by Izon qEV single SEC. The UEVs isolated by different methods showed high variability in both concentration and size besides a remarkable decrease between pre and post purified samples. Based on these results the most efficient and cheap method to isolate UEVs is the Izon qEV single SEC column.

Methods Summary: Four UEVs isolation techniques and Izon SEC purification were compared. Urine samples were collected and processed to discharge cellular debris. UEVs were isolated by Norgen, Exo Quick, QIAGEN, AMICON and Izon SEC methods. All samples were characterized in size and concentration by qNano instrument before and after protein purification. The protein concentration was evaluated by Pierce TM BCA assay. Based on samples concentration and protein contamination we identified the most efficient method to isolate extracellular vesicles in urine.

Keywords: Urinary Extracellular Vesicles; Biomarkers Isolation; Purification; Tunable Resistive Pulse Sensing Technology
\end{abstract}

\section{Abbreviation}

EVS: Extracellular Vesicles; UEVS: Urinary Extracellular Vesicles; MVS: Multivesicular; Mirna: Micro RNA; SEC: Size Exclusion Column; TRPS: Tunable Resistive Pulse Sensing Technology; PSD: Particle Size Distribution

\section{Introduction}

Recently, the clinical and scientific interest in the study of extracellular vesicles (EVs) has been increased due to their potential role as noninvasive biomarkers of pathophysiological health status in different medical areas. This small membrane bound vesicles are secreted from MVs-multivesicular body (exosome) in the extracellular space, or directly released from the cell through plasma membrane fusion and exocytosis (microvesicles) [1]. Their identity and characterization is not well defined, although there are many studies that focus on their lipidomic $[2,3]$, proteomic $[4,5]$, and transcriptomic profiling [6,7], to exploit their possible role as biomarkers in different human pathologies.

These nanoparticles are involved in intercellular communication [8] and in many different biological processes such as: regulation of immune response, tumor proliferation, inflammatory response and antigen presentation [9,10]. EVs are present in various type of body fluids (e.g. blood, serum, saliva, milk and urine) [11] and their concentration, cellular origin, composition, function is body health-(state) dependent [10]. Particularly, EVs content is useful to understand in which physiological regulation process they are involved. Many studies have shown that EVs carry different type of cargo, such as micro RNA (miRNA) and proteins. miRNA are short single-strand RNA molecules that are involved in several biological processes including cell death, proliferation, apoptosis, fat metabolism and oncogenesis [12]. Thus, measuring the concentration, cellular origin and content of EVs in body fluids has also a 
potential to provide novel and non-invasive disease biomarkers. In the nephrological field the study of urinary extracellular vesicles (UEVs) have acquired a great importance, because they can directly reflect the healthy status of the kidney [13]. Therefore, UEVs are suitable for the prevention of kidney rejection in the transplanted population. In the last few years several methods and techniques for the isolation and characterization of UEVs have been developed, that are cheaper and more effective than the already known ultracentrifugation method. The choice of the best method to isolate and identify UEVs is essential to obtain good quality samples and optimal concentrations. Thus, in this study, we compared five different methods (four UEVs isolation techniques and Size Exclusion Column (SEC)) to clarify which among these is the most convenient. The evaluation of the five different procedures was done based on concentration, size and protein contamination of the isolated UEVs.

\section{Material and Methods}

Preparation of samples

Early morning fresh urine samples were collected from healthy volunteers and promptly processed as describe by Felix R., et al [14]. Informed consent was obtained from all individuals included in this study. The samples were used to isolate and characterize UEVs, whereas the samples excess was stored at $-80^{\circ} \mathrm{C}$. The storage at $-80^{\circ} \mathrm{C}$ compared with $+4{ }^{\circ} \mathrm{C}$ or $-20^{\circ} \mathrm{C}$, better prevent urine nanoparticles degradation, although freshly processed urine is the most favorable approach [15].

UEVs were isolated from $5 \mathrm{ml}$ of processed urine using five different methods: 1) Norgen Urine Exosome RNA Isolation Kit 2) Exo Quick-TC $^{\text {TM }}$ Exosome Precipitation Solution 3) QIAGEN exo Easy Maxi Kit, 4) Izon qEV single Size Exclusion Column and 5) the ultracentrifugation procedure (by Amicon ${ }^{\circledR}$ filter column).

\section{Urine extracellular vesicles isolation methods}

Method 1 (Norgen Urine Exosome RNA Isolation Kit; Norgen Biotek Corp, Ontario, CA, USA): According to Norgen protocol, described in Rachel E., et al. [16], this kit was adapted to isolate EVs from urine samples.

At the end of the procedure the supernatant, containing the eluted UEVs, was collected for further analysis.

Method 2 (ExoQuick-TCTM Exosome Precipitation Solution; System Biosciences, Mountain View, CA, USA): Urine samples were added Exo Quick-TC solution and processed as report in the protocol [17]. Afterwards, pellet was suspended in phosphate-buffered saline (PBS).
Method 3 (QIAGEN exo Easy Maxi Kit; QIAGEN, Leipzig GmbH, Hilden, GE): The QIAGEN protocol adopted in Daniel Enderle., et al. [18] was modified (as described in QIAGEN handbook) to isolate extracellular vesicles from urine samples. The same volume of XBP QIAGEN Buffer and urine sample were placed in a new tube and reversed for five or six times. The samples were left at room temperature for 15 minutes then transfer to a QIAGEN filter column and centrifuged at $500 \mathrm{~g} \times 1 \mathrm{~min}$. The flow-through was discarded and the filter column inserted in a new tube. We added XWP QIAGEN Buffer and centrifuged for $3000 \mathrm{~g}$ x $5 \mathrm{~min}$ to wash UEVs. Afterwards, the flow-through was discarded and vesicles were eluted with XE QIAGEN Buffer (centrifuged at $500 \mathrm{~g}$ and after at $3000 \mathrm{~g} \mathrm{x}$ $5 \mathrm{~min})$.

Method 4 (Ultracentrifugation procedure by Amicon ${ }^{\circledR}$ filter column; Billerica, Massachusetts, USA): The protocol described in Andrea Del Fattore., et al. [19] has been modified to ultrafiltration only (to maintain the integrity and functionality of the EVs) and applied to urine samples for vesicle isolation.

Method 5 (qEVsingle Size Exlusion Column; Izon Science, Burnside, Christchurch, NZ): Urine samples were mixed before processing. qEV columns (Analytical-scale max sample volume_100 $\mu \mathrm{L}$ ) were left at room temperature and the buffer was pipetted out above the top filter. $100 \mu \mathrm{l}$ of urine sample were loaded and $200 \mu \mathrm{l}$ of fraction were collected. The first five fractions are the "void volume" which does not contain vesicles. Immediately after the void volume, the following three fractions were collected. These are the fractions that contain UEVs and were processed further.

\section{Quantification and characterization of UEVs}

The UEVs extracted by the five methods were characterized by their quantity and diameter size. To obtain these data, the tunable resistive pulse sensing technology (TRPS) of the Izon qNanoTM (Izon Science, Burnside, Christchurch, NZ) was considered. The TRPS is the most appropriate and accurate technique to determine the particle size distribution (PSD) of extracellular vesicles [20].

Izon qNano measures continuously the electrical resistance of a small pore filled with conductive medium that is in contact with the solution containing the EVs [21,22]. Around the pore is applied an electric current needed to measure the resistance of the pore. Whereas the vesicles are moving through the pore, the pore resistance increase. This resistive pulse is revealed by Izon software as a decrease of current and it is detected as a particle with a definite size diameter [23]. To distinguish resistive pulses from noise, the threshold is set below the noise by the analysis software. The 
maximum particles size that qNano can detect is directly correlated with nanopore size. Particles that are larger than pore size cause the vesicles flow blockage and consequently lead to an inadequate particles count. In the present study we adopted pore with an exclusion size of $150 \mathrm{~nm}$, NP150. Quantification was performed based on calibrator CPC200 (Diameter $210 \mathrm{~nm}$ ) with a particle rate concentration of $1 \times 10^{9}$. The instrument has been set with the following parameters: current flow between $110-130 \mathrm{~mA}$, pore stretch $47 \mathrm{~mm}$, pressure $20 \mathrm{mbar}$ and current voltage $0.58 \mathrm{~V}$.

\section{Results and Discussion}

Results

Urinary extracellular vesicles are possible novel elective biomarkers of kidney health state. Indeed, UEVs carry different type of cargo such as protein and miRNA, that are suitable biomarkers released from renal cells. The isolation and detection of these vesicles may provide a useful diagnostic tool for early detection of kidney diseases. There are several methods for isolation and characterization of UEVs from urine samples collected from human patients, however up to now, a gold-standard technique for isolating UEVs in clinical practice has not be identified. Furthermore, many of the actual procedures to isolate vesicles from urine show protein contamination, that can interfere with particles analysis and quantification leading to a wrong assessment. In our study, we compared different methods for UEVs isolation and analyzed the resulting concentration, particle size pre and post purification, and protein contamination. Our aim was to describe in detail the most efficient and convenient method to isolate UEVs. The isolation methods adopted in this study are: QIAGEN exoR Neasy serum/plasma maxi kit, Norgen Exosome RNA Isolation kit, SDB ExoQuick-TC ${ }^{\mathrm{TM}}$, Amicon ${ }^{\circledR}$ Ultrafiltration column and Izon qEVsingle Size Exclusion Column. The samples isolated through the first four kits were also purified by Izon qEVsingle Size Exclusion Column to remove possible proteins contamination.

To detected the UEVs concentration and size we considered Tunable Resistive Pulse Sensing technology (TRPS) by Izon qNano. The instrument showed different pre purification values of UEVs' concentration with a range between $1.27 \times 10^{8}-4.38 \times 10^{9}$ (Figure 1; Table 1). The highest score for the isolated particles was reached by Amicon ${ }^{\circledR}$ Ultrafiltration method whereas the QIAGEN was not quantifiable, probably due to the interference between QIAGEN reagents and the nanopore (NP150) used for qNano quantification. The same evaluation was performed also after the SEC purification. The highest amount of particles was shown by Izon qEVsingle Size Exclusion Column (6.47 x 108) in the directly purified urine samples. Although this data showed a great throughput for SEC purifier samples, the ultrafiltration followed by SEC purification

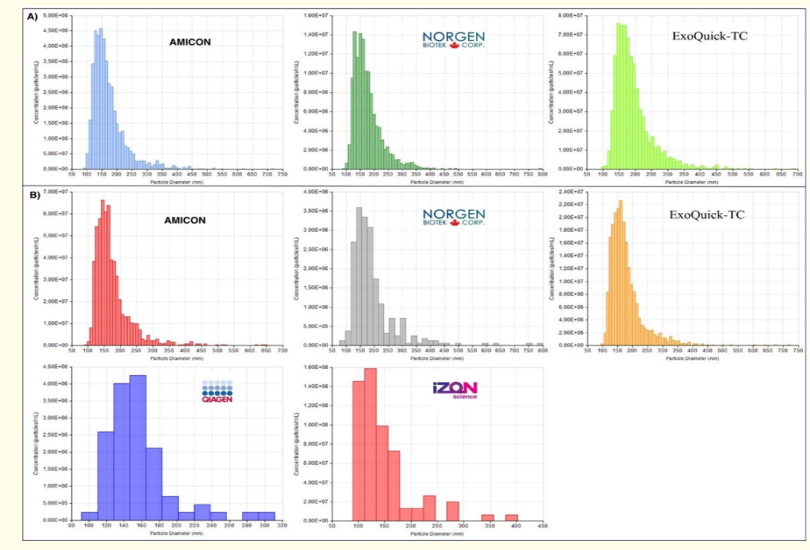

Figure 1: The size distribution of UEVs pre (A) and post (B) purification shows a similar distribution with the high concentration in the range of the microvesicles area.

resulted to be a satisfying method too, able to reach a concentration rank of $5.96 \times 10^{8}$. The size distribution of UEVs isolated by these different methods is within the macrovesicles size range (more than $100 \mathrm{~nm}$ ) [24], both for pre and post purified samples. The particle diameter distribution was between 123 - 161 nm (Figure 1; Table 1). All pre and post purified samples were analyzed to reveal possible protein contamination, by Pierce ${ }^{\mathrm{TM}}$ BCA Protein assay (Thermo Fisher scientific). The measurement showed a great variability in protein concentration by the different methods with the best performance obtained by Izon qEVsingle Size Exclusion Column. However, all aliquots treated by Izon columns showed a protein contamination between $0.2-0.35 \mathrm{mg} / \mathrm{ml}$ (Table 2).

\section{Discussion}

More than ten years have passed from the first reports on urinary vesicles [25] and currently a lot of methods for extracellular vesicles isolation are present, all based on different approaches such as: ultracentrifugation, density gradients, polymer-based precipitation, microfiltration and size-exclusion techniques. All of them sustain a high quality of their procedures considering vesicles concentration, size and purity [26]. Based on these considerations we tried to identify the best isolation method, among those listed, taking in account the criteria listed above and at the same time the reproducibility in laboratory. To our knowledge this is the first time that five different methodologies (four technique and direct SEC purification) are performed, at the same time, to identify the best approach for UEVs isolation considering both protein contamination and laboratory enforceability.

Our results showed differences in vesicles size and concentration (Figure 1; Table 1). At first glance, our study showed that the 


\begin{tabular}{|l|c|c|c|c|c|}
\hline $\begin{array}{c}\text { Urine } \\
\text { Volume }\end{array}$ & Isolation Technique & $\begin{array}{c}\text { UEVs size } \\
\text { diameter (Pre- } \\
\text { Purification) }\end{array}$ & $\begin{array}{c}\text { UEVs raw } \\
\text { concentration (Pre- } \\
\text { Purification) }\end{array}$ & $\begin{array}{c}\text { UEVs raw } \\
\text { UEVs size diameter } \\
\text { (Post- Purification) }\end{array}$ & $\begin{array}{c}\text { concentration } \\
\text { (Post- } \\
\text { Purification) }\end{array}$ \\
\hline $5 \mathrm{ml}$ & $\begin{array}{c}\text { QIAGEN } \\
\text { exoRNeasy serum/ } \\
\text { plasma maxi kit }\end{array}$ & Not detectable & Not detectable & 156 & $1.53 \times 10^{7}$ \\
\hline $5 \mathrm{ml}$ & $\begin{array}{c}\text { Norgen Exosome RNA } \\
\text { Isolation kit }\end{array}$ & 131 & $1.27 \times 10^{8}$ & 146 & $1.93 \times 10^{7}$ \\
\hline $5 \mathrm{ml}$ & $\begin{array}{c}\text { SDB Exo } \\
\text { Quick-TCTM }\end{array}$ & 143 & $7.51 \times 10^{8}$ & 161 & $2.16 \times 10^{8}$ \\
\hline $5 \mathrm{ml}$ & $\begin{array}{c}\text { Amicon Ultrafiltration } \\
\text { column }\end{array}$ & 147 & $4.38 \times 10^{9}$ & 145 & $5.96 \times 10^{8}$ \\
\hline $5 \mathrm{ml}$ & $\begin{array}{c}\text { Izon qEVsingle } \\
\text { Size Exclusion Column }\end{array}$ & & & 123 & $6.47 \times 10^{8}$ \\
\hline
\end{tabular}

Table 1: UEVs concentrations and size distribution pre and post Izon SEC protein purification.

\begin{tabular}{|l|c|c|}
\hline \multicolumn{1}{|c|}{ UEVs Isolation Technique } & $\begin{array}{c}\text { Protein concentration [mg/ml]_ } \\
\text { Pre-purification }\end{array}$ & $\begin{array}{c}\text { Protein concentration [mg/ml]_ } \\
\text { Post-purification }\end{array}$ \\
\hline QIAGEN exoRNeasy serum/plasma maxi kit & 0.7 & 0.25 \\
\hline Norgen Exosome RNA Isolation kit & 8.4 & 0.32 \\
\hline SDB ExoQuick-TCTM & 23 & 0.31 \\
\hline Amicon Ultrafiltration column & 11.47 & 0.35 \\
\hline Izon qEVsingle Size Exclusion Column & & 0.2 \\
\hline
\end{tabular}

Table 2: UEVs protein contaminations pre and post Izon SEC purification.

best method to isolate urinary vesicles was Amicon ${ }^{\circledR}$ Ultrafiltration with a vesicles concentration of $4.38 \times 10^{9}$, whereas the one with the disputable performance was the Norgen Exosome RNA Isolation kit with a raw concentration of $1.27 \times 10^{8}$. In this first phase the QIAGEN kit did not give detectable results probably due to the interference of its reagents with the Izon qNano instrument. This first analysis did not take into account the possible protein contamination of isolated particles. However, their graphic distribution was homogeneous and their concentration-peak was within the area of interest of the extracellular vesicles (Figure 1A). In all case, the highest sample concentration after isolation was in a diameter size range between 131 and $147 \mathrm{~nm}$ that is within the range of macrovesicles size. Analyzing post-purified samples, we could observe that particles raw concentration dropped down, but it was still possible to quantify it. After purification also QIAGEN processed samples showed a good range of UEVs concentration although the best performance was observed for samples directly purified by Izon SEC column. The homogeneity and distribution of detected particles show the same kind of particles despite their spread distribution. Indeed, these vesicles belong to the same group, as shown in pre purified samples, that is the cluster of macrovesicles (Figure 1B). The presence of protein contamination in the different methods considered, confirm a great variability, especially between pre and post purification. The greatest protein contamination was detected in the samples processed by Exo Quick kit (23 mg/ml). Whereas, QIAGEN kit showed a low level of proteins contamination between pre and post purification $(0.7-0.25 \mathrm{mg} /$ $\mathrm{ml}$ ). However, the best performance was observed for Izon SEC purification that show the lowest amount of protein among all the methods considered. It has to be pointed out that the difference in protein concentration in the post purified samples is negligible (about $0,15 \mathrm{mg} / \mathrm{ml}$ ). The distribution of pre and post-purification particles is also homogeneous and is found in the microvesicles area.

This comparative study provides a starting point for a broader research on novel possible reliable and noninvasive kidney damage biomarkers. In our study Izon qEV single SEC resulted to be the most efficient method to isolate and purify urine extracellular vesicles, that are suitable for NGS and Proteomics analysis. This 
method allows to have a greater quantity of traceable particles and without protein contamination, therefore the ideal condition for evaluating the real concentration of EVs in biological fluids.

Considering that urine is the unique biological fluid that shows a great variability $(\mathrm{pH}$, concentration, composition and osmolarity of dissolved solutes) even in the same individual and in the different time of the day, it would be interesting to extend the samples analysis. Sampling urine at several hours of the day on healthy volunteers versus patients with various types of kidney disease will be useful to understand how the variation of functional and clinical data lead to a different expression of urinary extracellular vesicles.

\section{Author Contributions}

AC and SN conceived the study, collected samples and wrote the manuscript. AC performed UEVs isolation and purification. MG and AMT performed qNano analysis and BCA dosages. GF and DM collected samples. PDG, GP and LM contributed to the manuscript revision. All the authors have accepted responsibility for the entire content of this submitted manuscript and approved submission.

\section{Financial and competing interests disclousure}

This study was supported by the charity Il Sogno Di Stefano ONLUS. The funding organization(s) played no role in the study design; in the collection, analysis, and interpretation of data; in the writing of the report; or in the decision to submit the report for publication.

\section{Ethical Approval}

All procedures performed in studies involving human participants were in accordance with the ethical standards of the institutional and/or national research committee and with the $1964 \mathrm{Hel}-$ sinki declaration and its later amendments or comparable ethical standards. This article does not contain any studies with animals performed by any of the authors.

\section{Bibliography}

1. Raposo G and Stoorvogel W. "Extracellular vesicles: exosomes, microvesicles, and friends". Journal of Cell Biology 200.4 (2013): 373- 383.

2. Llorente A., et al. "Molecular lipidomics of exosomes released by PC-3 prostate cancer cells". Biochimica et Biophysica Acta 1831 (2013) 1302-1309.

3. Skotland T., et al. "Lipids in exosomes: Current knowledge and the way forward". Progress in Lipid Research 66 (2017): $30-41$.
4. Pisitkun T., et al. "Identification and proteomic profiling of exosomes in human urine". Proceedings of the National Academy of Sciences of the United States of America 101 (2004): 13368-13373.

5. Gonzales PA., et al. "Large-scale proteomics and phosphoproteomics of urinary exosomes". Journal of the American Society of Nephrology 20 (2009): 363-379.

6. Miranda KC., et al. "Nucleic acids within urinary exosomes/ microvesicles are potential biomarkers for renal disease". Kidney International 78 (2010):191-199.

7. Schageman J., et al. "The complete exosome workflow solution: from isolation to characterization of RNA cargo". BioMed Research International (2013): 253957.

8. Camussi G., et al. "Exosomes/microvesicles as a mechanism of cell-to-cell communication”. Kidney International 78 (2010): 838- 848.

9. Xiao D., et al. "Identifying mRNA, microRNA and protein profiles of melanoma exosomes". PLoS One 7 (2012): e46874.

10. Yuana Y., et al. "Extracellular vesicles in physiological and pathological conditions". Blood Reviews 27 (2013): 31- 39.

11. Linares R., et al. "High-speed centrifugation induces aggregation of extracellular vesicles". Journal of Extracellular Vesicles 4 (2015): 29509.

12. Soltaninejad E., et al. "Differential expression of microRNAs in renal transplant patients with acute T-cell mediated rejection". Transplant Immunology 33 (2015): 1-6.

13. Salih M., et al. "Urinary extracellular vesicles and the kidney: biomarkers and beyond". American Journal of Physiology-Renal Physiology 306 (2014): F1251-F129.

14. Royo F., et al. "Different EV enrichment methods suitable for clinical settings yield different subpopulations of urinary extracellular vesicles from human samples". Journal of Extracellular Vesicles 5 (2016): 29497.

15. Oosthuyzen W., et al. "Quantification of human urinary exosomes by nanoparticle tracking analysis". The Journal of Physiology 591 (2013): 5833- 5842.

16. Crossland RE., et al. "Evaluation of optimal extracellular vesicle small RNA isolation and qRT-PCR normalisation for serum and urine". Journal of Immunological Methods 429 (2016): 3949. 
17. Alvarez ML., et al. "Comparison of protein, microRNA, and mRNA yields using different methods of urinary exosome isolation for the discovery of kidney disease biomarkers". Kidney International 82 (2012): 1024-1032.

18. Enderle D., et al. "Characterization of RNA from Exosomes and Other Extracellular Vesicles Isolated by a Novel Spin Column-Based Method". PLoS One 10 (2015): e0136133.

19. Del Fattore A., et al. "Immunoregulatory Effects of Mesenchymal Stem Cell-Derived Extracellular Vesicles on T Lymphocytes". Cell Transplant 24 (2015): 2615- 2627.

20. van der Pol E., et al. "Particle size distribution of exosomes and microvesicles determined by transmission electron microscopy, flow cytometry, nanoparticle tracking analysis, and resistive pulse sensing". Journal of Thrombosis and Haemostasis 12 (2014): 1182- 1192.

21. Ito T., et al. "Comparison of nanoparticle size and electrophoretic mobility measurements using a carbon-nanotubebased coulter counter, dynamic light scattering, transmission electron microscopy, and phase analysis light scattering". Langmuir 20 (2004): 6940-6945.

22. DeBlois RW., et al. "Electrokinetic measurements with submicron particles and pores by the resistive pulse technique". Journal of Colloid and Interface Science 61 (1977): 323- 335.

23. Coumans FAW., et al. "Reproducible extracellular vesicle size and concentration determination with tunable resistive pulse sensing". Journal of Extracellular Vesicles 3 (2014): 25922.

24. Merchant ML., et al. "Isolation and characterization of urinary extracellular vesicles: implications for biomarker discovery". Nature Reviews Nephrology 13 (2017): 731-749.

25. Thongboonkerd V., et al. "Proteomic analysis of normal human urinary proteins isolated by acetone precipitation or ultracentrifugation". Kidney International 62 (2002): 14611469.

26. Van Deun J., et al. "The impact of disparate isolation methods for extracellular vesicles on downstream RNA profiling". Journal of Extracellular Vesicles 3 (2014).

Volume 3 Issue 11 November 2019

(C) All rights are reserved by Andrea Carraro., et al. 